\title{
The research of the single row piles designing in deep foundation pit
}

\author{
Nan Lv ${ }^{1, a^{*}}$, Hui Min Li \\ ${ }^{1,2}$ Xi'an University of Architecture and Technology, Xi'an ,China \\ alvn75@126.com
}

Keywords: deep foundation pit,single row pile,single fulcrum,equivalent beam method,bolt Abstract. Single fulcrum row pile retaining its unique advantages in deep foundation excavation has been widely used. In this paper, the design with equivalent beam method of single fulcrum row piles in deep foundation pit retaining structure was analyzed.It will provide technical support for the construction.

In recent years, our various types of construction and civil engineering have developed rapidly. High-rise.High-rise buildings in large numbers, the underground structures housing, construction, municipal engineering, underground garage, subway stations and others in excavation of foundation pit are essential in the construction. In order to ensure the foundation pit construction and the main body of the underground structure security, the environment intact, certain measures of support will be taken.

The effect of supporting structures in foundation pit during digging are soil retaining, water retaining, ensuring the foundation pit excavation and infrastructure construction safely and smoothly, and no damages are caused during foundation construction to adjacent buildings, roads and underground pipelines, etc.

In deep foundation pit supporting engineering,row pile support has its unique characteristics, so has been widely used in foundation pit supporting [1]. Specific include:

A. Row pile construction technology is simple, low cost. The layout is flexible;

B. Compared with underground continuous wall, the row pile had poor anti-seepage and integrity;

C. Building envelope of Non-Driven row pile compared with prefabricated sheet pile, have advantages of no-noise, no-vibration, no-compaction, etc.

Row piles are generally applicable to medium depth $(6 \sim 10 \mathrm{~m})$ pit supporting, but in recent years are also applied in the excavation within $20 \mathrm{~m}$ depth of foundation pit, as shown in figure 1,2 as follows. Single fulcrum row pile buried depth is deeper. Through setting bolt or internal supporting at the fulcrum, the pile is suffered horizontal force.The force control the horizontal displacement of the pile body,reduce the depth. In foundation pit supporting, because of its reasonable force form, single fulcrum of single pile has been widely applied in engineering practice.

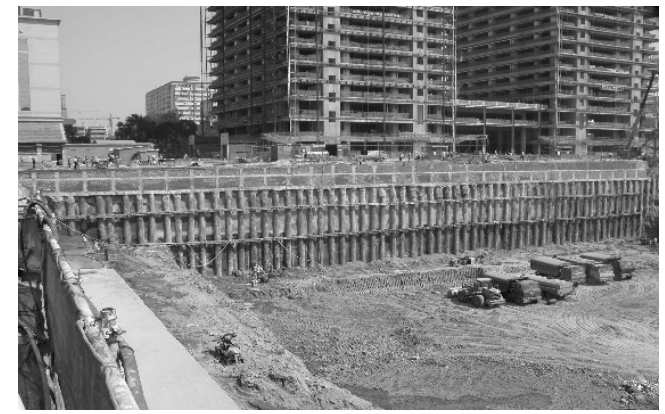

Fig.1 Row pile construction

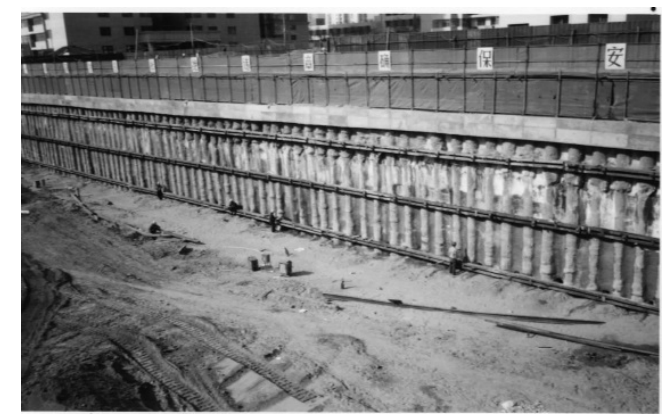

Fig.2 Row pile construction

\section{The basic principle of equivalent beam method}

The calculation method of the single fulcrum row pile supporting are: balance method, graphic 
method, the equivalent beam method, m method, the finite element method, etc. The force of pile in supporting is shown in figure 3. As a method of calculating the internal force of maintenance structure ,equivalent beam method is applicable to the maintenance structure with support. The method is easy to calculate, so it is widly used in engineering practice [2].

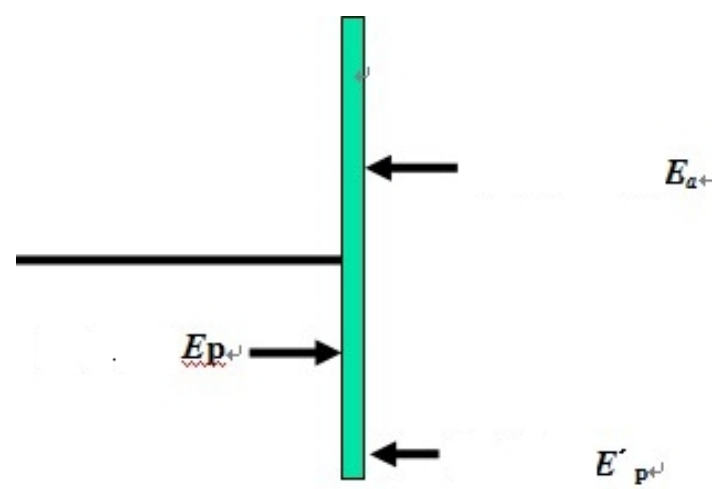

Fig.3 force analysis of pile in supporting

$\mathrm{Ea}$-active earth pressure

Ep_— passive earth pressure

$\mathrm{E}^{\prime} \mathrm{p}$ _ passive earth pressure

Calculation principle of equivalent beam method [3] is: when the pile drilling soil deep, pile bottom suffers the clamping effect of soil,pile head suffers the supporting of bolt,and central pile suffers soil compaction. a mechanical balancing system is made in the whole system. Pile stress analysis in Equivalent beam calculation is shown in figure 4.

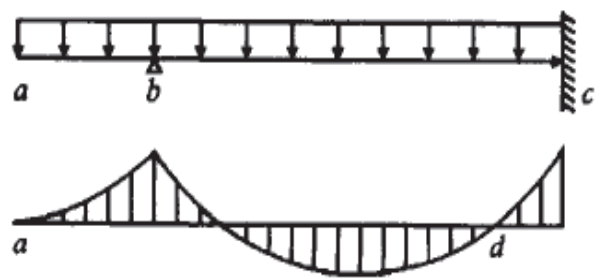

Fig. 4 pile stress analysis in Equivalent beam calculation

In figure 4 , beam ac at point $\mathrm{c}$ is the fixed end, at point $\mathrm{b}$ is hinged to stand.In bending moment diagram, point $d$ is the inflection point of deflection line. If beam ac is broken at $d$, it beccoms a beam ad by setting a free supporting at $d$. Under the same distribution of load, bending moment of beam ad will remain the same, so we take beam ad as the equivalent beam of beam ac. So pile can be divided into two parts, the top is simply supported beam, the bottom is statically indeterminate beam. We can use the method of structural mechanics to calculate the internal force of the pile.

In the calculation of equivalent beam method, the position of the inflection point (bending moment is equal to zero) will be determined at first. The point of zero bending moment underground is very close to the point of zero earth pressure.In order to simplify the calculation, we take the point of zero earth pressure as the inflection point.

\section{Calculation steps}

Using Rankine's theory or coulomb theory, we can calculate the earth pressure strength acting on the pile, draw the pressure profile, as shown in figure 5. The Rankine's theory with clear concept and simple formula, can be calculated directly whether for clayey soil or cohesionless soil .It is widely used in engineering.But the friction effect between the filling and the wall is not considered.It leads to increase of the active earth pressure and decrease of passive earth pressure . Coulomb theory cannot be directly used to calculate the earth pressure of clayey soil.The result of the active earth pressure is satisfy the engineering precision, but the deviation value of passive earth pressure deviation is bigger.

we will determine the position of the inflection point and calculate the distance y from the ground to 
the inflection point. At the point $y$, the active earth pressure and passive earth pressure pile is equal. That is:

$$
y=\frac{\gamma K_{a} H-2 c \sqrt{K_{a}}+q K_{a}-2 c \sqrt{K_{p}}}{\gamma\left(K_{p}-K_{a}\right)}
$$

$\gamma$ - unit weight $(\mathrm{kN} / \mathrm{m} 3)$;

$c$ - cohesive force $(\mathrm{kPa})$;

$K_{a}$-active earth pressure

$K_{p}$ - passive earth pressure

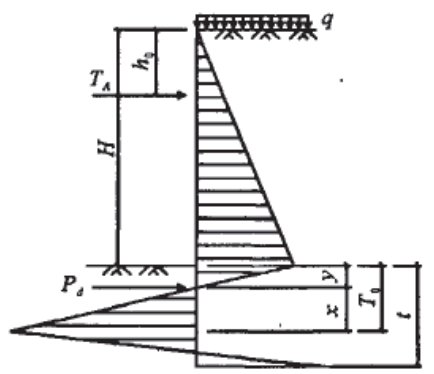

Fig.5 Earth pressure distribution

According to the simply supported beam, we can calculate the maximum bending moment Mmax of equivalent beam and two fulcrum reaction forces, $T_{A}$ and $P_{d}$.

Calculating minimum capacity of the pile $t_{0}$.

$$
t_{0}=y+x
$$

according to the principle that the moment of Pd and passive earth pressure acting at the bottom of pile are equal, we can calculate $\mathrm{X}$, namely:

$$
\begin{aligned}
& p_{d} x=\frac{1}{6} \gamma\left(K_{p}-K_{a}\right) x^{3} \\
& x=\sqrt{\frac{6 P_{d}}{\gamma\left(K_{p}-K_{a}\right)}}
\end{aligned}
$$

The actual embedded depth of pile bottom must be under $\mathrm{x}$. Pile capacity $t=K t_{0} \quad \mathrm{~K}$ is $1.1 \sim 1.2$ 。 That is:

$$
t_{0}=y+\sqrt{\frac{6 P_{d}}{\gamma\left(K_{p}-K_{a}\right)}}
$$

When the equivalent beam method are used to calculate more fulcrum pile (anchor), the basic principle and calculation steps are similar to single fulcrum equivalent beam method. There are Multi-span continuous beam on the inflection point of the more protection equivalent beam.Calculation of counteracting force and pile bending moment will be In accordance with the analysis of continuous beam using the method of structural mechanics. Calculation of pile capacity also can use the calculate steps of the equivalent beam method of the single fulcrum pile.

Using equivalent beam method for pile design is a kind of simple and effective method.It can ensure the strength and stability of deep foundation pit supporting structure.It make the construction process quality and safety. 


\section{References}

[1] LIU Guo-bin,WANG Wei-dong.Foundation Engineering Handbook[M].beijing:China Building Industry Press, 2009.

[2] ZHU Li-xia. Supporting Structure Design of Foundation Pit with the Equivalent Beam Method[M]. Soil Eng.and Foundation, 2010,24(6):42-45.

[3] XIE Meng,HOU Ke-peng,FU He-lin.Application of the Equivalent Beam Method in Design of A Deep Pit[J].Soil Eng.and Foundation, 2008,22(1):14-17.

[4] Technical code for building foundation pit JGJ120-99.

[5] ZHANG Hui,GUO Miwen.Application of Soldier Piles with a Single Fulcrum in the Deep Foundation Pit [J]. Geoteehnical Engineering Technique, 2011,25(3): 120-124.

[6] REN Li-mei.Flexible Single-row Pile Design Calculations[J]. Construction \& Design for Project,2010,(05): 108-110.

[7] WANG Fei.The Stress Analysis of the Deep Foundation Pit Single Row Piles Supporting Structure[M]. Shanxi Science \& Technology of Communications ,2014,(06): 61-64. 